\title{
La tecnología en la disyuntiva familiar en tiempos de pandemia por COVID-19, 2020
}

\author{
Technology in the family dilemma in times of COVID-19 pandemic, 2020
}

\author{
María Mercedes Macías-Cedeño', (D) (8) María Dolores Chávez-Vera² (D) (8)
}

\author{
Cómo citar \\ Macías-Cedeño, M. y Chávez-Vera, M. (2021). La tecnología en la disyuntiva familiar en tiempos de pandemia COVID-19 \\ 2020. Socialium, 5(2), 55-71. https://doi.org/10.26490/uncp.sl.2021.52.919
}

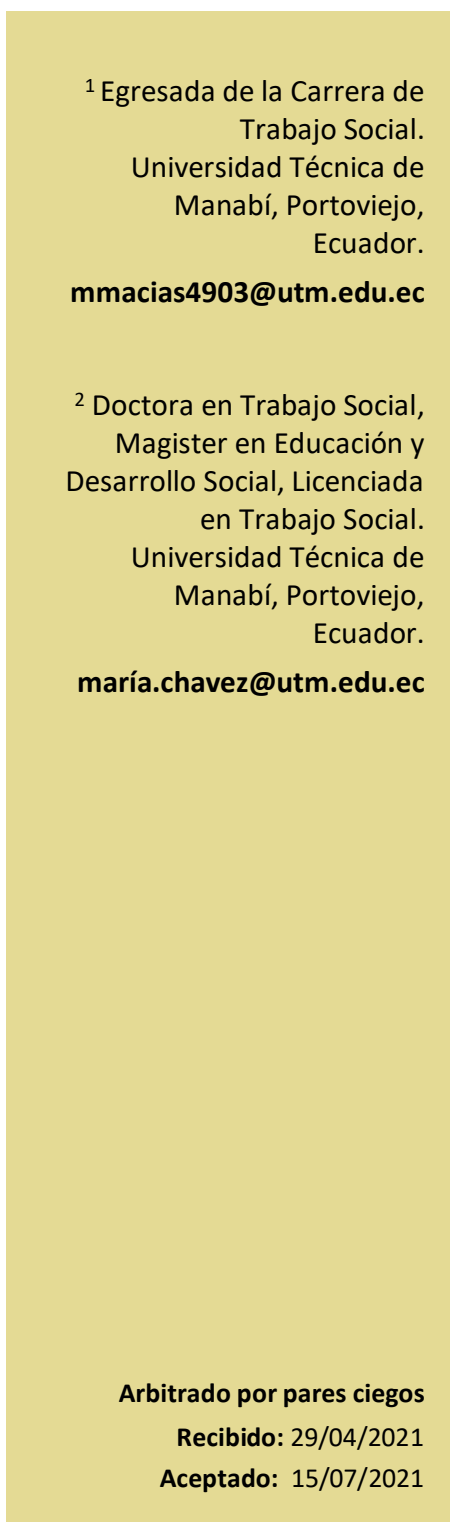

\section{RESUMEN}

La tecnología es una herramienta que aporta a la sociedad, mantiene al emisor y receptor comunicados y aprendiendo constantemente, subrayando no ser la excepción durante la pandemia COVID-2019, ha permitido a las familias estar informadas sobre las situaciones acontecidas, de igual manera hizo frente al entretenimiento para acceder a redes sociales, juegos en línea, educación virtual y teletrabajo; por tal motivo, el objetivo de estudio permite analizar las horas, problemas en el uso de equipos tecnológicos en padres, madres e hijos; identificar las percepciones de los efectos sobre el uso de la tecnología durante la pandemia COVID-19; asociar el número de horas del uso del celular con el castigo en los hijos. Se realizó una investigación cuantitativa no experimental, descriptivo de corte transversal. La muestra estuvo conformada por 50 padres familias entre las edades de 23 a 55 años $(M=34,58$ DT=9,738). Se aplicó dos cuestionarios uno sociodemográfico y Prácticas Parentales de Robinson. Los resultados revelaron que el equipo tecnológico usado con mayor frecuencia por los integrantes de la familia es el celular. Las percepciones de los efectos cuando se hace uso de la tecnología mostraron conductas de enojo, fatiga y rebeldía, también existieron problemas de comunicación, mala relación entre padres e hijos, aislamiento y falta de autoridad por parte de los padres. Se concluye que el padre, madre e hijos permanecen entre 2 a 4 horas conectados al celular siendo una causa probable de desencadenar problemas como deterioró la convivencia familiar y la relación de pareja.

Palabras clave: tecnología; horas; familia; conductas; estilos parentales; pandemia COVID-19.

\section{ABSTRACT}

Technology is a tool that contributes to society. It keeps the sender and the receiver communicated and constantly learning. It has not been an exception during the COVID-19 Pandemic, indeed. It has allowed communication between families about all the arousing circumstances. It has also been the mean to access entertainment such as social networks, online games, virtual education and telework; these are some of the reasons to have as objectives: to analyze the hours, problems in the use of technological equipment in father, mothers and children; identify perceptions of the effects on the use of technology during the COVID-19 Pandemic; associate the number of hours of cell phone use with punishment for children. A nonexperimental, descriptive, cross-sectional quantitative investigation was performed. The sample consisted of 50 family parents aged between 23 to 55 years $(M=34,58 S D=9,738)$. A sociodemographic and a Robinson's Parental Practices questionnaire were applied. The result reveals that telephones are the most frequently used technological equipment among the family members. Behaviors of anger, fatigue and rebellion, communication problems, poor relationship between parents and children, isolation and lack of parents' authority were the perceptions of the effects of technology use. It can be concluded that father, mother and children remain connected to cell phones between 2 to 4 hours, being a probable cause of triggering problems, such as decline of family coexistence and the couple relationship.

Keywords: technology; hours; family; behaviors; parenting styles; COVID-19 pandemic. 


\section{Introducción}

El uso desmedido de la tecnología en general se ha convertido en una lucha constante dentro de la sociedad, sin embargo investigaciones realizadas por los autores (Samada, 2020; Rodríguez et al., 2019) indican que los equipos tecnológicos utilizados con mayor frecuencia dentro del hogar son el televisor, computadoras y el teléfono celular, resaltando este último, el equipo más usado y de fácil traslado, otras investigaciones resaltaron que la consola de video juegos sobresale en el uso diario por partes de los hijos (Díaz et al., 2020).

Otras literaturas indican que los hombres hacen mayor uso de las herramientas tecnológicas en relación a las mujeres, destacando que ambos tienen preferencia en utilizar el celular, ya que a través de este equipo pueden comunicarse, obtener cierta información, y tener acceso a redes sociales como Facebook y WhatsApp, esta última se resalta con un uso frecuente (González et al., 2020; Pedrero et al., 2018).

En cuanto al uso que los hijos le dan a las TIC dentro del grupo familiar (Nobles et al., 2016 ; Carrasco et al., 2017) manifiestan que una parte de ellos se dedican frecuentemente a jugar Minecraft a través del celular, así mismo observan videos o escuchan música en la plataforma de YouTube, a su vez hacen uso de las redes sociales como Facebook y WhatsApp, siendo esta una actividad que todos realizan dentro de casa incluyendo a los padres, con la finalidad de chatear con amigos, seguidos a esto el uso que le dan al computador es menor, debido a que solo lo utilizan para tareas académicas. Cabe resaltar que todas estas situaciones ocurren cuando no existe un control dentro del hogar, por tal razón, Lepincnik y Samec (2013) argumentan que los hijos deben tener un uso limitado en los medios tecnológicos, por el peligro que estos representan.

Según Sánchez et al. (2018) determinan que la supervisión practicada por los padres de familia se basa en normas de conexión debido a que existen altas posibilidades que los hijos accedan a página de contenido adulto.

De acuerdo a la frecuencia en el uso de los equipos tecnológicos varios autores llegaron a una misma conclusión destacando que la herramienta más utilizada por padres e hijos es el teléfono móvil con un uso de 3 a 5 horas diarias, por lo que este equipo se convierte en un accesorio indispensable y de necesidad (Luque y Herrera, 2019; Nery y Chávez, 2020).

En consecuencia, investigaciones realizadas por Díaz et al. (2019) enfatizan que pasar muchas horas en equipos tecnológicos desvincula a los más pequeños a relacionarse con el entorno social. Sin 
embargo, desde la posición de Carrasco et al. (2017) el uso frecuente del celular está vinculado al enojo y grito en los niños cuando pierden un juego.

Las dificultades que se presencian por el abuso de la tecnología son diversos, por tanto, Pedrero et al. (2018) determina que tanto hombres y mujeres sufren desgastes emocionales, como el estrés debido al uso desmedido en acceder a las redes sociales, en cambio para Rodríguez et al. (2019) añaden que los problemas que presentan los adolescentes en el hogar o en aulas de clases son de concentración, ya que no dedican tiempo a realizar tareas del hogar y académicas, por lo tanto esto podría conducir a un problema en el núcleo familiar.

Por otro lado, estudios afirman que los medios tecnológicos son sinónimos de desunión familiar tal como lo argumenta Barrera y Duque (2014) quienes sostienen que la comunicación parental se ha visto afectada, porque los hijos permanecen mayor tiempo en el mundo digital, ocasionando distancia, y para lograr un diálogo con ellos es a través de las redes sociales. Además, González et al. (2018) vincula a las relaciones conyugales que atraviesan momentos de controversia debido al uso prolongado de las tecnologías mientras se está con la pareja, disminuyendo momentos de intimidad, como una cena, ocasionando malos entendidos, discusiones y celos.

No obstante, se destaca que las tecnologías no son perjudiciales en su totalidad todo dependerá del uso y el control que se le otorgue (Hernández et al., 2014), por tal motivo Yubero et al. (2018) manifiesta sobre la mediación parental que existen dentro de la familia, pues aquí se habla sobre los beneficios y lo perjudicial que es la tecnología si no se establecen medios estratégicos de uso. Así mismo en las investigaciones realizadas por Bartau et al. (2018) resaltan que los padres emplean tácticas de retirar el celular como medio de castigo. En cambio, otros practican un estilo librenegociador, debiéndose a que los padres tienen estudios superiores (Sánchez et al., 2017).

Ahora bien, el uso de equipos tecnológicos en momentos de pandemia COVID-2019 permitió a las familias permanecer informadas y comunicadas a través de celulares, computadora, Tablet, de igual forma ayudaron a que se mantengan comunicadas con otros familiares que viven en otras ciudades (Prada et al., 2020). Para Arámburo et al. (2020) indican que las TIC permitieron que alumnos reciban clases por diferentes plataformas tecnológicas como Zoom. También se destaca que padres de familia realizaron Teletrabajo durante el confinamiento, pero no todos tenían el dominio de las herramientas tecnológicos (Rosario et al., 2020).

Para ello los propósitos del presente trabajo fueron:

Analizar las horas, problemas familiares y la relación de pareja en el uso equipos tecnológicos. 
Identificar las percepciones de los efectos sobre el uso de la tecnología en pandemia COVID-19. Asociar el número de horas en el uso del celular en pandemia COVID-19 con el castigo en hijos.

\section{Método}

Tipo de estudio: Para la realización del proceso de investigación se utilizó un diseño no experimental de carácter descriptivo de corte transversal, apoyado en un enfoque cuantitativo, a nivel empírico se utilizó la técnica de la encuesta estructurada para la recopilación de datos, el cuestionario de preguntas cerradas con escala Likert. La unidad de análisis estuvo caracterizada por padres y madres de familias de las manzanas 1 y 2 de la comunidad El Naranjo de la Parroquia Urbana Simón Bolívar del cantón Portoviejo.

Población y muestra: La población objeto de la investigación es de 700 habitantes, por lo cual la muestra estuvo constituida por 50 padres de familias (5 padres y 20 madres) de la manzana 1 y (5 padres y 20 madres) de la manzana 2 de la comunidad urbana El Naranjo del Cantón Portoviejo de la Provincia de Manabí de la República del Ecuador. El rango estuvo constituido entre la edad de 23 a 55 años ( $M=34,58 \mathrm{DT}=9,738)$. El nivel de estudio estuvo representado por el $8 \%$ primaria, $50 \%$ secundaria, $22 \%$ presentan estudios universitarios y un $20 \%$ aún están estudiando la Universidad. En relación al tipo de vivienda, el 8 \% corresponden a los encuestados que viven en casas de caña, el $66 \%$ de hormigón y un $26 \%$ las viviendas son mixtas.

\section{Instrumentos de recolección de datos:}

Cuestionario sociodemográfico: Se elaboró un cuestionario sociodemográfico en relación a los objetivos planteados dentro de este estudio, en total de 22 preguntas fueron elaboradas por las autoras. Cabe indicar que el cuestionario estructurado incluye variables como, 1) Datos personales; (sexo, edad, grupo a investigar, manzana a las que pertenece, instrucción, y tipo de vivienda); 2) Quién y para qué hacen uso de equipos tecnológicos; 3) Frecuencia sobre el uso de los equipos; 4) Percepciones de los efectos de conlleva el uso de equipos tecnológicos dentro de la familia y otros, cabe indicar que las preguntas están enmarcadas a la emergencia del COVID-19. La mayoría de las preguntas son de tipo Likert que van desde muy frecuente, frecuentemente, ocasionalmente, raramente y nunca.

El Cuestionario de Prácticas Parentales: (Parenting Practices Questionnarie), elaborado por Robinson et al. (1995). Consta de un total de 62 preguntas tipo Likert que van desde 1 (nunca) a 
5(siempre). Mide tres tipologías 1 autoridad (subescala: afecto 11 ítems, razonamiento 7 ítems, democrática 5 ítems y fácil de llevar 4 ítems); 2 autoritarias (subescalas: hostilidad 4 ítems, castigo corporal 6 ítems, no razonamiento/punitivo 6 ítems, 4 Directividad autoritaria); 3 permisivas (subescalas: falta de supervisión 6 ítems, mala conducta 4 ítems, autoconfianza 5 ítems).

Procedimiento de la recolección de datos: La aplicación de los cuestionarios sociodemográficos y Práctica Parentales de Robinson se lo realizó con 50 padres de familias de la Manzanas 1 y 2 de la comunidad urbana "El Naranjo". La aplicación de la encuesta fue de carácter confidencial y se la llevó a cabo de manera presencial, respetando todos los protocolos de protección de bioseguridad debido a la pandemia, el proceso de rellenar los formularios se les manifestó a los padres de familias objetivo de estudio. El tiempo duración en aplicar la encuesta fue de 30 minutos a 35 minutos aproximadamente por padres y su culminación en completar todos los formularios fue de 8 días.

Análisis de los datos: Para analizar los datos se utilizó la plataforma del software IBM SPSS Statistics versión 22. Se realizó el análisis estadístico descriptivo de todas las variables a través de (tablas contingencias); en primer lugar se observó las variables sociodemográficas que aparecen en la tabla 1, en la tabla 2 se observó número de horas con equipos tecnológicos; en la tabla 3 se realizó un análisis entre la frecuencia del uso de la tecnología y problemas durante la pandemia COVID-19; tabla 4 relación entre la frecuencia del uso de la tecnología en horas de la comida del padre, madre e hijos; tabla 5 se observa las percepciones de los efectos en el uso de la tecnología en tiempos de COVID19; se observa la frecuencia entre el deterioro de la convivencia familiar, preferencia en el uso de la tecnología en detrimento de tareas del hogar, ignora a los miembros de la familia, deterioro de la relación de la pareja; y por último en la tabla 7 se observa (Chi-cuadrado Chi-square) para obtener una asociación entre las Escala Permisiva (falta de supervisión) de las Practicas Parentales de Robinson entre castigo frecuente al hijo y horas de uso del teléfono del celular 


\section{Resultado}

\section{Tabla 1}

Características Sociodemográficas

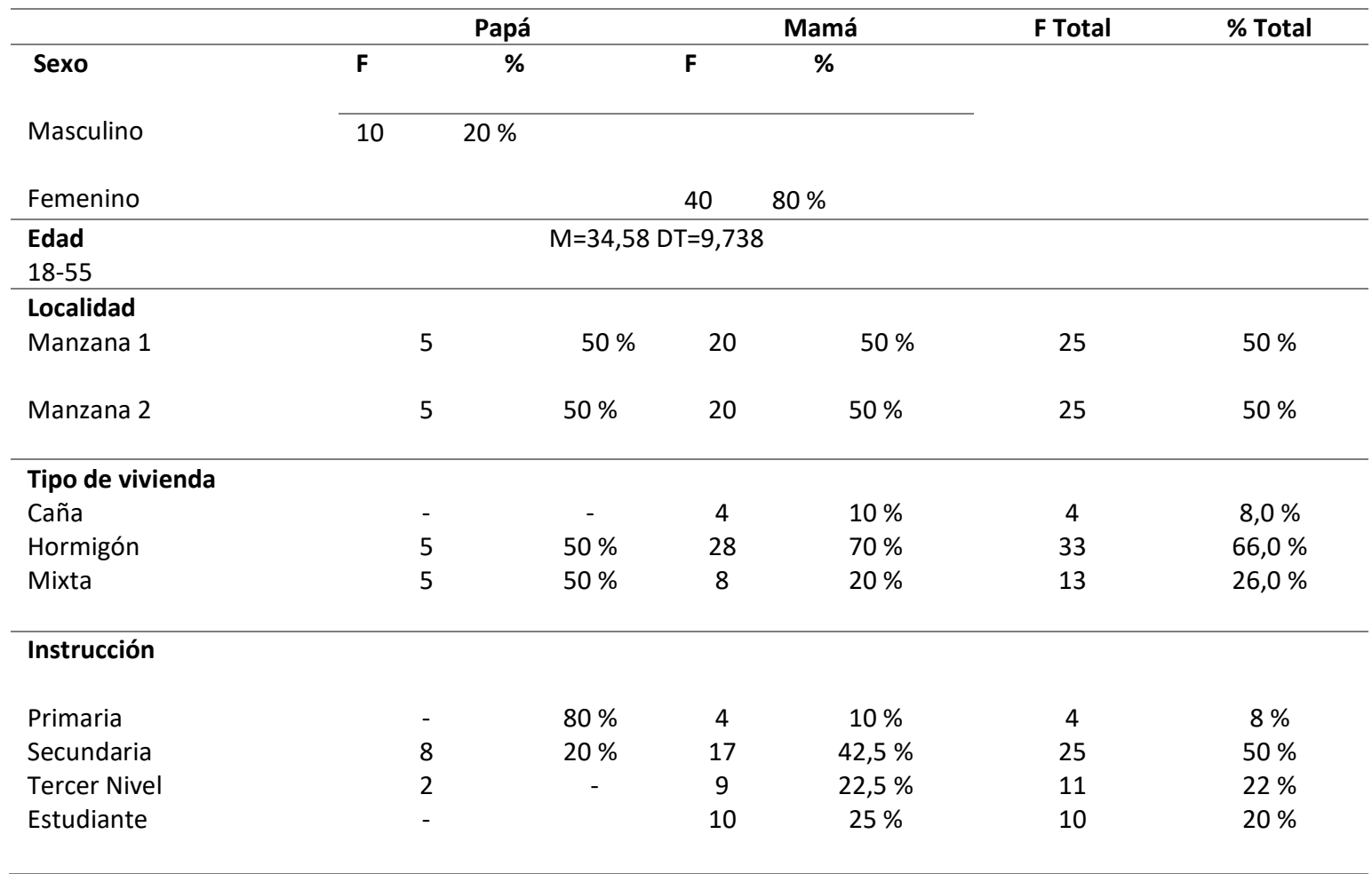

Nota. Tomado de los Padres de familias de las Manzanas 1 y 2 de la comunidad El Naranjo (2021).

La tabla 1 refleja que la mayoría de los padres de familias encuestados son mujeres con un $80 \%$ $(n=40)$, frente un $20 \%(n=10)$ fueron hombres, la edad promedio reflejada fue de 18 a 55 años $(\mathrm{M}=34,58 \mathrm{DT}=9,738)$. En relación al tipo de vivienda, un porcentaje elevado corresponden al $66 \%$ ( $n=33$ ) viven en casas de hormigón. De acuerdo a la instrucción un $8 \%(n=4)$ han logrado la primaria, un $50 \%$ ( $n=25)$ secundaria, un $22 \%$ ( $n=11$ ) de los padres tienen título de tercer nivel, y un $20 \%(n=10)$ aún estudian. 


\section{Tabla 2}

Frecuencia y porcentajes sobre las horas en uso de equipos tecnológicos entre padres, madres e hijos

\begin{tabular}{|c|c|c|c|c|c|c|c|c|c|c|c|c|c|c|c|c|c|c|c|c|c|c|c|c|c|c|c|c|c|c|c|c|c|}
\hline & \multicolumn{11}{|c|}{ Padres } & \multicolumn{11}{|c|}{ Madres } & \multicolumn{11}{|c|}{ Hijos } \\
\hline & \multicolumn{11}{|c|}{ Horas } & \multicolumn{11}{|c|}{ Horas } & \multicolumn{11}{|c|}{ Horas } \\
\hline & \multicolumn{5}{|c|}{ Padre } & \multicolumn{4}{|c|}{ Madre } & & & \multicolumn{5}{|c|}{ Padre } & \multicolumn{4}{|c|}{ Madre } & & & \multicolumn{5}{|c|}{ Padre } & \multicolumn{4}{|c|}{ Madre } & \multirow[b]{2}{*}{ 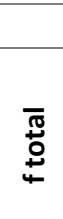 } & \multirow[b]{2}{*}{$\begin{array}{l}\overline{\widetilde{J}} \\
\stackrel{0}{*} \\
\stackrel{0}{\circ}\end{array}$} \\
\hline & $\begin{array}{l}2 \\
\mathrm{~h}\end{array}$ & $\begin{array}{l}3 \\
h\end{array}$ & $\begin{array}{l}4 \\
\mathrm{~h}\end{array}$ & $\begin{array}{l}\frac{\text { 믕 }}{0} \\
\text { 응 } \\
\text { 으 }\end{array}$ & $\begin{array}{l}1 \\
\mathrm{~h}\end{array}$ & $\begin{array}{l}2 \\
h\end{array}$ & $\begin{array}{l}3 \\
\mathrm{~h}\end{array}$ & $\begin{array}{l}4 \\
\mathrm{~h}\end{array}$ & 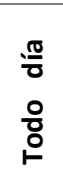 & 胥 & $\begin{array}{l}\bar{\pi} \\
\stackrel{0}{0} \\
\stackrel{0}{\circ}\end{array}$ & $\begin{array}{l}1 \\
\mathrm{~h}\end{array}$ & $\begin{array}{l}2 \\
\mathrm{~h}\end{array}$ & $\begin{array}{l}3 \\
h\end{array}$ & $\begin{array}{l}4 \\
\mathrm{~h}\end{array}$ & $\begin{array}{l}1 \\
\mathrm{~h}\end{array}$ & $\begin{array}{l}2 \\
\mathrm{~h}\end{array}$ & $\begin{array}{l}3 \\
\mathrm{~h}\end{array}$ & $\begin{array}{l}4 \\
\mathrm{~h}\end{array}$ & 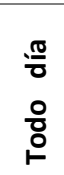 & 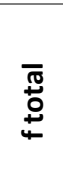 & 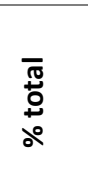 & $\begin{array}{l}1 \\
\mathrm{~h}\end{array}$ & $\begin{array}{l}2 \\
\mathrm{~h}\end{array}$ & $\begin{array}{l}3 \\
\mathrm{~h}\end{array}$ & $\begin{array}{l}4 \\
\mathrm{~h}\end{array}$ & $\begin{array}{l}1 \\
\mathrm{~h}\end{array}$ & $\begin{array}{l}\mathbf{2} \\
\mathrm{h}\end{array}$ & $\begin{array}{l}3 \\
\mathrm{~h}\end{array}$ & $\begin{array}{l}4 \\
\mathrm{~h}\end{array}$ & $\begin{array}{l}\frac{\pi}{0} \\
\circ \\
\stackrel{0}{0} \\
\circ\end{array}$ & & \\
\hline $\begin{array}{l}\text { Equipos } \\
\text { tecnológicos }\end{array}$ & & & & & & & & & & & & & & & & & & & & & & & & & & & & & & & & & \\
\hline Celular & 4 & - & 6 & 2 & - & 5 & 17 & 12 & 4 & 50 & $100 \%$ & - & 4 & - & 6 & - & 2 & 8 & 21 & 9 & 50 & $100 \%$ & & 5 & 5 & - & 7 & $\begin{array}{l}1 \\
3\end{array}$ & 9 & 8 & 3 & 50 & $100 \%$ \\
\hline Computadora & 5 & 3 & 2 & - & 3 & 7 & 6 & 2 & - & 28 & $56 \%$ & - & - & 2 & - & 2 & 5 & 7 & 2 & - & 18 & $36 \%$ & 2 & 5 & - & - & 3 & 6 & 3 & - & - & 19 & $38 \%$ \\
\hline Tv & - & 3 & 2 & - & 13 & $\begin{array}{l}1 \\
6\end{array}$ & - & 3 & - & 37 & $74 \%$ & 6 & - & 2 & 2 & 20 & 10 & 2 & - & - & 42 & $84 \%$ & - & 3 & 2 & - & 15 & 7 & 7 & - & - & 34 & $68 \%$ \\
\hline Tablet & - & - & 2 & - & - & - & - & - & - & 2 & $4 \%$ & - & - & 2 & - & - & - & - & - & - & 2 & $4 \%$ & 5 & - & - & - & 2 & - & 3 & 2 & - & 12 & $24 \%$ \\
\hline $\begin{array}{l}\text { Consola de } \\
\text { Videos juegos }\end{array}$ & 2 & - & - & - & - & 3 & - & - & - & 5 & $10 \%$ & - & & 2 & - & - & - & - & - & - & 2 & $4 \%$ & - & 2 & - & 2 & 4 & - & 2 & - & - & 10 & $20 \%$ \\
\hline
\end{tabular}

En la tabla 2 el $100 \%(n=50)$ de los padres de familias manifestaron que los hijos hacen uso constante del celular, así mismos añadieron que los papás lo utilizan en un período de 2 a 3 horas diarias y las mamás entre 3 a 4 horas en el día. En los resultados se observa que ambos padres indican que la madre es quién hace mayor uso de este equipo tecnológico. 


\section{Tabla 3}

Frecuencia y porcentajes de los problemas y uso de equipos tecnológicos durante la pandemia COVID-19

\begin{tabular}{|c|c|c|c|c|c|c|c|c|c|c|c|c|}
\hline & \multicolumn{6}{|c|}{ Uso de la tecnología /COVID-19 } & \multicolumn{6}{|c|}{ Problemas en el uso de tecnología/ COVID-19 } \\
\hline & \multicolumn{2}{|c|}{ Padre } & \multicolumn{2}{|c|}{ Madre } & \multirow[b]{2}{*}{$\begin{array}{c}f \\
\text { tota } \\
\text { I }\end{array}$} & \multicolumn{3}{|c|}{ Padre } & \multicolumn{2}{|c|}{ Madre } & \multirow[b]{2}{*}{$\begin{array}{c}\mathbf{f} \\
\text { total }\end{array}$} & \multirow[b]{2}{*}{$\begin{array}{c}\% \\
\text { total }\end{array}$} \\
\hline & $f$ & $\%$ & $f$ & $\%$ & & $\begin{array}{c}\% \\
\text { tota } \\
\text { I }\end{array}$ & $f$ & $\%$ & f & $\%$ & & \\
\hline $\begin{array}{l}\text { Muy } \\
\text { frecuentemente }\end{array}$ & 3 & $6 \%$ & 23 & $46 \%$ & 26 & $52 \%$ & 3 & $6 \%$ & 7 & $14 \%$ & 10 & $20 \%$ \\
\hline Frecuentemente & 5 & $10 \%$ & 15 & $30 \%$ & 20 & $40 \%$ & - & - & 7 & $14 \%$ & 7 & $14 \%$ \\
\hline Ocasionalmente & - & - & - & - & & & 7 & $14 \%$ & 9 & $18 \%$ & 16 & $32 \%$ \\
\hline Raramente & 2 & $4 \%$ & 2 & $4 \%$ & 4 & $8 \%$ & - & - & 6 & $12 \%$ & 6 & $12 \%$ \\
\hline Nunca & - & - & - & - & - & - & - & - & 11 & $22 \%$ & 11 & $22 \%$ \\
\hline
\end{tabular}

Nota. Tomado de los Padres de familias de las Manzanas 1 y 2 de la comunidad El Naranjo (2021).

Se observa en la tabla 3 que un $46 \%(n=23)$ de las madres expusieron que el uso de equipos tecnológicos por las familias en pandemia COVID-19 se dio de manera muy frecuentemente, correspondiente a los padres un $10 \%(n=5)$ añadieron que esta actividad se daba frecuente. En relación a los problemas suscitados por el uso, padres y madres en un $32 \%$ ( $n=16)$ indicaron la existencia de problemas dentro del hogar por el uso de la tecnología.

\section{Tabla 4}

Frecuencia y porcentaje del uso de la tecnología en horas de comida

\begin{tabular}{lccccccc}
\hline & & Padre & \multicolumn{3}{c}{ Madre } & & \\
\cline { 2 - 8 } & $\mathbf{f}$ & & $\%$ & 13 & $26 \%$ & 13 & $26 \%$ \\
\hline Padre & - & - & 2 & $4 \%$ & 2 & $4 \%$ \\
\hline Madre & - & - & 8 & $16 \%$ & 10 & $20 \%$ \\
\hline Hijo & 2 & $4 \%$ & 17 & $34 \%$ & 25 & $50 \%$ \\
\hline
\end{tabular}

Nota. Tomado de los Padres de familias de las Manzanas 1 y 2 de la comunidad El Naranjo (2021).

En la tabla 4 se atribuye que un $34 \%(n=17)$ de las madres y un $16 \%(n=8)$ de los padres encuestados declararon que ninguno de los miembros de la familia hace uso del celular en horas de comida, pero en un $26 \%$ ( $n=13$ ) de madres de familias puntualizaron que es el padre quién hace uso del móvil en esos momentos de familia. 


\section{Tabla 5}

Percepciones de los efectos en el uso de la Tecnología en tiempos de COVID- 19

\begin{tabular}{|c|c|c|c|c|c|c|}
\hline & \multicolumn{2}{|c|}{ Padres } & \multicolumn{2}{|c|}{ Madres } & \multirow[b]{2}{*}{$f$ total } & \multirow[b]{2}{*}{$\%$ total } \\
\hline Conductas & $f$ & $\%$ & $f$ & $\%$ & & \\
\hline Enojo & 3 & $6 \%$ & 16 & $32 \%$ & 19 & $38 \%$ \\
\hline Tranquilidad & - & - & 8 & $16 \%$ & 8 & $16 \%$ \\
\hline Fatiga & 2 & $4 \%$ & 3 & $6 \%$ & 5 & $10 \%$ \\
\hline Rebeldía & - & - & 5 & $10 \%$ & 5 & $10 \%$ \\
\hline Depresión & 3 & $6 \%$ & - & - & 3 & $6 \%$ \\
\hline Ninguno & 2 & $4 \%$ & 8 & $16 \%$ & 10 & $20 \%$ \\
\hline \multicolumn{7}{|l|}{ Problemas familiares } \\
\hline Aislamiento & - & - & 11 & $22 \%$ & 11 & $22 \%$ \\
\hline $\begin{array}{l}\text { Problemas de } \\
\text { comunicación }\end{array}$ & 5 & $10 \%$ & 18 & $36 \%$ & 23 & $46 \%$ \\
\hline $\begin{array}{l}\text { Problemas autoridad / } \\
\text { padres }\end{array}$ & - & - & 3 & $6 \%$ & 3 & $6 \%$ \\
\hline Discusiones / hijos & 5 & $10 \%$ & 8 & $16 \%$ & 13 & $26 \%$ \\
\hline
\end{tabular}

Nota. Tomado de los Padres de familias de las Manzanas 1 y 2 de la comunidad El Naranjo (2021).

Se evidencia en la tabla 5 las percepciones sobre los efectos que trae consigo el uso de la tecnología por parte de los integrantes de la familia los cuales presentan conductas de enojo $38 \%$ ( $n=19)$, fatiga $10 \%(n=5)$, rebeldía $10 \%(n=5)$, también indica la existencia de problemas de comunicación entre los integrantes de la familia en un $46 \%$ ( $n=23)$, seguido las discusiones de padres con hijos $26 \%(n=13)$, aislamiento $22 \%$ ( $n=11$ ) y problemas de autoridad por parte de los padres de familia $6 \%(n=3)$. Se observó que ambos padres de familias ubican al enojo como principal conducta por hacer uso de la tecnología, de forma parecida recalcan que el principal problema concurrente entre los integrantes es de comunicación. 


\section{Tabla 6}

Frecuencia y porcentajes sobre el uso de la tecnología en la convivencia familiar, preferencias, relación de la pareja

\begin{tabular}{|c|c|c|c|c|c|c|c|c|c|c|c|c|c|c|c|c|c|c|c|c|c|c|c|c|}
\hline & \multicolumn{6}{|c|}{ Deterioro de la convivencia familiar } & \multicolumn{6}{|c|}{$\begin{array}{l}\text { Preferencia en uso de la tecnología en } \\
\text { detrimento tareas del hogar }\end{array}$} & \multicolumn{6}{|c|}{$\begin{array}{l}\text { Ignora a miembros de la familia } \\
\text { (celular) }\end{array}$} & \multicolumn{6}{|c|}{$\begin{array}{c}\text { Deterioro de la relación de pareja } \\
\text { (celular) }\end{array}$} \\
\hline & \multicolumn{2}{|c|}{ Padre } & \multicolumn{2}{|c|}{ Madre } & \multirow[b]{2}{*}{$\begin{array}{c}f \\
\text { total }\end{array}$} & \multirow[b]{2}{*}{$\begin{array}{l}\% \\
\text { total }\end{array}$} & \multicolumn{2}{|c|}{ Padre } & \multicolumn{2}{|c|}{ Madre } & \multirow[b]{2}{*}{$\begin{array}{c}f \\
\text { total }\end{array}$} & \multirow[b]{2}{*}{$\begin{array}{l}\% \\
\text { total }\end{array}$} & \multicolumn{2}{|c|}{ Padre } & \multicolumn{2}{|c|}{ Madre } & \multirow[b]{2}{*}{$\begin{array}{c}f \\
\text { tot } \\
\text { al }\end{array}$} & \multirow[b]{2}{*}{$\begin{array}{l}\% \\
\text { total }\end{array}$} & \multicolumn{2}{|c|}{ Padre } & \multicolumn{2}{|c|}{ Madre } & \multirow[b]{2}{*}{$\begin{array}{c}f \\
\text { total }\end{array}$} & \multirow[b]{2}{*}{$\begin{array}{l}\% \\
\text { total }\end{array}$} \\
\hline & $f$ & $\%$ & $f$ & $\%$ & & & f & $\%$ & $f$ & $\%$ & & & $f$ & $\%$ & $\mathbf{f}$ & $\%$ & & & $f$ & $\%$ & f & $\%$ & & \\
\hline $\begin{array}{l}\text { Muy } \\
\text { frecuentemente }\end{array}$ & 2 & $4 \%$ & - & - & 2 & $4 \%$ & - & - & 5 & $10 \%$ & 5 & $10 \%$ & 3 & $6 \%$ & $\begin{array}{l}1 \\
2\end{array}$ & $24 \%$ & 15 & $30 \%$ & 5 & $10 \%$ & 3 & $6 \%$ & 8 & $16 \%$ \\
\hline Frecuentemente & 5 & $10 \%$ & 16 & $32 \%$ & 21 & $42 \%$ & 7 & $\begin{array}{l}14 \\
\%\end{array}$ & $\begin{array}{l}1 \\
9\end{array}$ & $38 \%$ & 26 & $52 \%$ & - & - & 6 & $12 \%$ & 6 & $12 \%$ & 3 & $6 \%$ & 2 & $4 \%$ & 5 & $10 \%$ \\
\hline Ocasionalmente & - & - & 8 & $16 \%$ & 8 & $16 \%$ & 3 & $6 \%$ & $\begin{array}{l}1 \\
6\end{array}$ & $32 \%$ & 19 & $38 \%$ & 7 & $14 \%$ & $\begin{array}{l}2 \\
2\end{array}$ & $44 \%$ & 29 & $58 \%$ & 2 & $4 \%$ & 24 & $48 \%$ & 26 & $52 \%$ \\
\hline Raramente & 3 & $6 \%$ & 10 & $20 \%$ & 13 & $26 \%$ & - & - & - & - & - & - & - & - & - & - & - & - & - & - & 11 & $22 \%$ & 11 & $22 \%$ \\
\hline Nunca & - & - & 6 & $12 \%$ & 6 & $12 \%$ & - & - & - & - & - & - & - & - & - & - & - & - & - & - & - & - & - & - \\
\hline
\end{tabular}

Nota. Tomado de los Padres de familias de las Manzanas 1 y 2 de la comunidad El Naranjo (2021).

La tabla 6 presenta otras situaciones ocurridas por el uso de la tecnología, los encuestados argumentaron en un $42 \%$ ( $\mathrm{n}=21$ ) el deterioro de los lazos familiares por pasar muchas horas usando la tecnología, un $52 \%$ ( $n=26)$ indicaron que sus hijos prefieren hacer uso de las TIC y no hacer actividades del hogar, un 58 \% ( $n=29)$ añadieron que por el uso del celular se ignora a las personas en conversaciones familiares y del mismo modo un 52 \% ( $=26)$ se ve afectada las relaciones de pareja. En relación a estas situaciones la madre indica que todas se dan con bastante frecuencia y desde el punto de vista del padre si se da, pero regularmente. 


\section{Tabla 7}

Asociación entre las variables horas de uso del celular y castigo frecuentemente a los hijos

\begin{tabular}{|c|c|c|c|c|c|c|}
\hline \multirow[b]{2}{*}{$\begin{array}{l}\text { Horas de uso del celular } \\
\text { en los hijos }\end{array}$} & \multicolumn{4}{|c|}{ Castigo frecuentemente a mi hijo } & & \multirow{2}{*}{$\begin{array}{c}\text { Pruebas de } \\
\text { Chi-cuadrado } \\
\text { Sig. asintótica }\end{array}$} \\
\hline & Nunca & $\begin{array}{l}\text { Pocas } \\
\text { veces }\end{array}$ & $\begin{array}{c}\text { Algunas } \\
\text { veces }\end{array}$ & & & \\
\hline & $f$ & $f$ & $f$ & $\begin{array}{c}\mathbf{f} \\
\text { total }\end{array}$ & $\%$ & \\
\hline 1hora & 7 & - & - & 7 & $14 \%$ & 000 \\
\hline 2 horas & 9 & 6 & 3 & 18 & $36 \%$ & 43,918 \\
\hline 3 horas & 7 & 7 & - & 14 & $28 \%$ & \\
\hline 4 horas & - & 8 & - & 8 & $16 \%$ & \\
\hline Todo el día & - & - & 3 & 3 & $6 \%$ & \\
\hline
\end{tabular}

Nota. Tomado de los Padres de familias de las Manzanas 1 y 2 de la comunidad El Naranjo (2021).

Se observa en la tabla 7 una asociación significativa $p<.005$ entre las variables horas que pasan los hijos en el celular con la variable castigo frecuentemente a los hijos, comprobándose una concordancia perfecta entre ellas.

\section{Discusión}

En primer lugar, los resultados obtenidos reflejan que tanto padre, madre e hijo hacen uso del celular frecuentemente, los hijos lo utilizan entre dos a tres horas en el día, y los padres lo hacen de tres a cuatros horas, estos hallazgos tienen discrepancia con la investigación realizada por los autores Besoli et al. (2018) quienes resaltan que los padres de familias presumen que sus hijos hacen uso del celular treinta minutos diarios con la finalidad de chatear con sus amigos y jugar, en cuanto a los padres hacen uso del mismo de una hora para consultar algún tipo de información y revisar las redes sociales.

Manteniéndose en el hecho del uso del celular otras investigaciones resaltan que hacer uso constante del teléfono móvil causa dependencia, del mismo modo a las aplicaciones que se utilicen dentro de él (Pedrero et al., 2018), en la misma línea se añade que los adolescentes pasan horas chateando por WhatsApp desencadenando un uso problemático al momento de usar el celular (Díaz et al., 2019).

El uso de equipos tecnólogos fue utilizado en la pandemia COVID-19 muy frecuentemente, lo que ocasionó problemas familiares dentro de los hogares. Estos datos tienen concordancia a hechos revelados por Pedrero et al. (2018) alegan que el uso prolongado de equipos tecnológicos causan en las personas problemas de mala conducta social, y el deterioro de las emociones, de esta forma resultados con similitud expuestos por (Matamala, 2016; Sánchez, et al., 2018 ; Arciniega, 2019) 
indican que los hijos no realizan las tareas encomendadas por el colegio debido a que pasan mucho tiempo en el celular, Tablet, consola o computador ocasionando que pierdan la noción del tiempo. A estas argumentaciones se contraponen la de los autores Hernández et al. (2014) donde se evidencia que parte de los encuestados declararon no existir problemas por el uso de equipos tecnológicos o por pasar horas conectados al celular o en consolas de video juegos.

Un $26 \%$ manifestaron que es el padre $(n=13)$ quien utiliza el celular en horas de la comida, según Samada (2017) argumenta que ambos proyectan malos hábitos de conductas hacia los hijos y esto se debe a que los padres no respetan las horas de comida, ni mucho menos espacios de conversación por estar pendiente de los teléfonos celulares. Otros datos obtenidos expresan que los padres prefieren pasar momentos en familia y de ocio con sus hijos (Muñoz y Bowen, 2019).

De acuerdo a las percepciones sobre los efectos del uso de la tecnología dentro del hogar los resultados reflejan que los integrantes presentan conductas de enojo, fatiga y rebeldía, cuando hacen uso de los equipos tecnológicos. No obstante, hallazgos similares argumentan que la falta de control, agresividad física y verbal son indicios que presentan los adolescentes cuando pasan horas prolongadas usando la tecnología (Bartau et al., 2018; Tur et al., 2019), en la misma línea ilustraciones llevadas a cabo por Dogliotti et al. (2020) destacan que los adolescentes están deprimidos, inquietos o nerviosos cuando no tienen acceso a la tecnología.

Al mismo tiempo se percibe problemas de comunicación, discusiones con los hijos, aislamiento y problemas de autoridad por parte de los padres. Por tanto, literaturas parecidas agregan que el uso de dispositivos móviles infiere en la comunicación directa debido a que las personas se muestran limitados en demostrar sus sentimientos (Nobles et al., 2016; Pari, 2019). En relación a la falta de autoridad, investigaciones similares resaltan que el poco conocimiento que tienen los padres en el manejo de las TIC, incide en que los hijos no cumplan las normas establecidas sobre el uso de la tecnología (Bartau et al., 2018). En cuanto a las discusiones entre padres e hijos, estudios aumentaron que el origen de las controversias se da por la distracción de los hijos en el celular (Peñuela et al., 2014).

Hay que precisar también, que en momentos de pandemia COVID-19 se deterioró la convivencia familiar y se prefirió hacer uso de la tecnología negándose a realizar tareas del hogar, a la vez se excluyó algún familiar por hacer uso del celular y esto también influyó en la relación de la pareja. En consonancia a este apartado Carrasco et al. (2017) explican que el uso de la tecnología ha disminuido momentos en familia, seguido a que los adolescentes han dejado de practicar algún tipo de deporte 
(Díaz et al., 2019). Estudios exponen que las personas se sienten molestos y desplazados cuando no le prestan atención (Peñuela et al., 2014). Del mismo modo se encontró que subir fotos a las redes sociales o comentar publicaciones a terceros es un motivo de discusión y de celos en la pareja (Vásquez y Pastor, 2018; Nery y Chávez, 2020).

\section{Conclusión}

Los resultados de la investigación evidenciaron que en la pandemia COVID-19 dentro de las familias pondero de manera muy frecuente el uso de equipos tecnológicos entre ellos el celular con una frecuencia de 2 a 4 horas diarias, siendo una causa de probabilidad de desencadenar problemas.

Las situaciones encontradas fueron que el padre es quién utiliza el celular en horas de comida, siendo una probabilidad de repeticiones en patrones de conducta hacia los demás miembros de la familia, así mismo se pone en manifiesto que las conductas presentadas por los integrantes del hogar es el enojo, fatiga y rebeldía, además, se evidenciaron otras percepciones en pandemia COVID-19 como problemas de comunicación, mala relación entre padres e hijos, aislamiento y la falta de autoridad por parte de los padres.

De acuerdo a los resultados descriptivos obtenidos de la investigación, existe un deterioro en la convivencia familiar, los hijos descartan las responsabilidades del hogar por hacer uso de la tecnología, del mismo modo el uso del celular es una acción que hace ignorar a los demás en conversaciones familiares, además afecta la relación conyugal. También se evidencia una asociación positiva entre las variables escogidas.

Debido a la poca muestra que representó el número de padres, los resultaron tuvieron cierta similitud con las respuestas de las madres, puesto a que en la mayoría de las tablas presentadas se observó concordancia con los datos obtenidos.

\section{Referencias}

Arámburo, M., Gandar, L., Medina, R. y Tirado, K. (2020). El impacto de las herramientas informáticas en el aprendizaje durante la pandemia. Revista ReDTIS, 4 (4), 17-24. https://www.redtis.org/index.php/Redtis/article/view/69/62

Arciniega-Carrión, A. (2019). Comunicación familiar y medios tecnológicos: Siglo XXI. Polo del Conocimiento, 4 (8), 23-36. http://dx.doi.org/10.23857/pc.v4i8.1041 
Barrera, V. y Duque, L. (2014). Familia e internet: consideraciones sobre una relación dinámica. Revista Virtual Universidad Católica del Norte, 41, 30-44. http://revistavirtual.ucn.edu.co/index.php/RevistaUCN/article/view/463/985

Bartau-Rojas, I., Aierbe-Barandiaran, A. y Oregui-González, E. (2018). Mediación parental del uso de Internet en el alumnado de Primaria: creencias, estrategias y dificultades. Comunicar, 26(54), 71-79. https://doi.org/10.3916/C54-2018-07

Besoli, G., Palomas, N. y Chamarro, A. (2018). Uso del móvil en padres, niños y adolescentes: Creencias acerca de sus riesgos y beneficios. Aloma, 36(1), 29-39. https://doi.org/10.51698/aloma.2018.36.1.29-39

Carrasco, F., Droguett, R., Huaiquil, D., Navarrete, A., Quiroz, M. y Binimelis, H. (2017). El uso de dispositivos móviles por niños: Entre el consumo y el cuidado familiar. Cuhso, 27(1), 108-137. https://cuhso.uct.cl/index.php/cuhso/article/view/1191

Díaz-López, A., Maquilón-Sánchez, J., y Mirete-Ruiz, A. (2020). Uso desadaptativo de las TIC en adolescentes: Perfiles, supervisión y estrés. Comunicar, 64 (28), 29-38. https://doi.org/10.3916/C64-2020-03

Díaz-Vicario, A., Mercader, C. y Gairín, J. (2019). Uso problemático de las TIC en adolescentes. Revista $\begin{array}{llll}\text { Electrónica de Investigación 21(7),1-11. } & \text { Educativa, }\end{array}$ https://doi.org/10.24320/redie.2019.21.e07.1882

Dogliotti, C., González, I., Botero, C. y Delfino, G. (2020). Uso y frecuencia de conexión a internet y bienestar subjetivo en jóvenes argentinos. Psicología Unemi, 4(7), 74-87. http://ojs.unemi.edu.ec/index.php/faso-unemi/article/view/1139

Hernández, M., López, P. y Sánchez, S. (2014). La comunicación en la familia a través de las TIC. $\begin{array}{llll}\text { Percepción de los } & \text { adolescentes. }\end{array}$ https://revistas.cardenalcisneros.es/index.php/PULSO/article/view/168

Gaxiola, J., Frías, M., Cuamba, N., Franco, J. y Olivas, L. (2006). Validación del cuestionario de prácticas parentales en una población mexicana. Enseñanza e Investigación en Psicología, 11(1),115128. https://www.redalyc.org/articulo.oa?id=2921110

González-Rivera, J., Segura-Abreu, L. y Urbistondo-Rodríguez, V. (2018). Phubbing en las Relaciones Románticas: Uso del Celular, Satisfacción en la Pareja, Bienestar Psicológico y Salud Mental. Interacciones, 4(2), 81-91. https://doi.org/10.24016/2018.v4n2.117 
González, A., Velázquez, L. y Grijalva, G. (2020). Viviendo con teléfono y pareja. Usos del celular en entornos de alta conectividad. Iztapalapa Revista de ciencias Sociales y humanidades, 89(2). 151-181. http://dx.doi.org/10.28928/ri/892020/aot3/gonzalezav/velazquezl/grijalvag

Lepicnik, J. y Samec, P. (2013). Uso de tecnologías en el entorno familiar en niños de cuatro años de Eslovenia. Comunicar, 40, 119-126. https://doi.org/10.3916/C40-2013-03-02

Luque-González, A. y Herrero-García, N. (2019). Impacto de la tecnología en la sociedad: El caso de Ecuador. Impacto de la tecnología en la sociedad: el caso de Ecuador. Universidad y Sociedad, 11(5), 176-182. https://rus.ucf.edu.cu/index.php/rus/article/view/1358

Matamala, C. (2016). Uso de las TIC en el hogar: Entre el entretenimiento y el aprendizaje informal. Estudios pedagógicos, 42(3), 293 311. https://dx.doi.org/10.4067/S071807052016000400016

Muñoz, N. y Bowen, B. (2019). La familia frente a las tecnologías de la información y comunicación (TIC) en adolescentes de la Unidad Educativa Gonzalo Escobar Barcia. Revista Caribeña de Ciencias Sociales. https://www.eumed.net/rev/caribe/2019/12/familia-tecnologias.html

Nery, S. y Chávez, B. (2020). Conflicto versus comunicación: Las redes sociales en la relación de pareja. $\begin{array}{llll}\text { Alternativas Cubanas en Psicología, 107-125. } & \text { 8(22), }\end{array}$ https://www.acupsi.org/articulo/288/conflicto-versus-comunicacin-las-redes-sociales-enla-relacin-de-pareja.html

Nobles, D., Londoño, L., Martínez, S., Ramos, A., Santa, G. y Cotes, A. (2016). Tecnologías de la comunicación y relaciones interpersonales en jóvenes universitarios. Revista Educación y Humanismo, 18(30), 14-27. https://doi.org/10.17081/eduhum.18.30.1311

Pari-Ccama, Y. (2019). Uso de los Smartphone en las relaciones familiares en estudiantes universitarios de la Universidad Nacional del Altiplano de Puno. Revista De Investigación en Comunicación Y Desarrollo, 10(2), 151-159. https://doi.org/10.33595/2226-1478.10.2.387

Pedrero, E., Ruiz, J., Rojo, G., Llanero, N., Pedrero, A., Morales, S. y Puerta, C. (2018). Tecnologías de la Información y la Comunicación (TIC): uso problemático de Internet, videojuegos, teléfonos móviles, mensajería instantánea y redes sociales mediante el MULTICAGE-TIC. Adicciones, 30 (1), 19-32. https://www.adicciones.es/index.php/adicciones/article/view/806

Prada-Núñez, R., Hernández-Suárez, A., y Maldonado-Estévez, A. (2020). Diagnóstico del potencial de las redes sociales como recurso didáctico en el proceso de enseñanza en época de 
$\begin{array}{llll}\text { aislamiento social. } & \text { Revista }\end{array}$ https://www.revistaespacios.com/a20v41n42/a20v41n42p22.pdf

Rosario-Rodríguez, A., Cruz-Santos, A. y González-Rivera, J. (2020). Demandas Tecnológicas, Laborales y Psicológicas del Teletrabajo durante la Pandemia por COVID-19. Revista Ciencias $\begin{array}{llll}\text { de la } & \text { Conducta, } & \text { 35(1), }\end{array}$ http://cienciasdelaconducta.org/index.php/cdc/article/view/33

Rodríguez, M., Díaz, M., Agostinelli, J. y Daverio, R. (2019). Adicción y uso del teléfono celular. Ajayu Órgano de Difusión Científica del Departamento de Psicología UCBSP, 17(2), 211-235. http://www.scielo.org.bo/scielo.php?script=sci_arttext\&pid=S2077$21612019000200001 \& \operatorname{lng}=e s \&$ tlng=es.

Robinson, C., Mandelco, B., Frost, S. y Hart, C. (1995). Authoritative, authoritarian, and permissive parenting practices: Development of a newmeasure. Psychological Reports, 77, 819-830. https://doi.org/10.2466/pr0.1995.77.3.819

Samada, Y. (2017). Influencia de las tecnologías de la información y la comunicación en las normas de convivencia familiar. Revista Electrónica Formación y Calidad Educativa (REFCaIE), 5(3), 87-98. http://www.refcale.uleam.edu.ec/index.php/refcale/article/view/1944

Sánchez-Valle, M., De Frutos-Torres, B. y Vázquez-Barrio, T. (2017). La influencia de los padres en la adquisición de habilidades críticas en Internet. Comunicar, 53, 103-111. https://doi.org/10.3916/C53-2017-10

Sánchez-Antolín, P., Andrés-Viloria, C. y Paredes-Labra, J. (2018) El papel de la familia en el desarrollo de la competencia digital. Análisis de cuatro casos. Digital Education Review, (34), 44-58. https://www.raco.cat/index.php/DER/article/view/348340

Tur-Porcar, A., Doménech, A. y Jiménez, J. (2019). Eficacia académica percibida, crianza, uso de internet y comportamiento en la adolescencia. Revista Latinoamericana de Psicología, 51(1), 38-47. http://dx.doi.org/10.14349/rlp.2019.v51.n1.5

Vásquez, N., y Pastor. (2018). Uso de redes sociales y mensajería instantánea en relaciones de pareja en la juventud: un estudio preliminar. Health and Addictions: Salud y drogas, 19 (1), 87-98. https://www.researchgate.net/publication/331021833 
Yuber, S., Larrañaga, E., Navarro, R. y Elche, M. (2018). Padres, hijos e Internet. Socialización familiar de la Red. Una relación compleja. Universitas Psychologica, 17(2), 1-13. https://doi.org/10.11144/Javeriana.upsy17-2.phis

\section{Contribución de los autores}

MMMC: Conceptualización, análisis formal, investigación, redacción: borrador original.

MDCHV: Metodología, supervición, validación, redacción: revisión y edición.

\section{Fuentes de financiamiento}

Autofinanciado.

\section{Conflictos de interés}

No presenta ningún conflicto de intereses.

\section{Correspondencia}

mmacias4903@utm.edu.ec 